\title{
Strongyloides stercoralis e strongiloidiasi in Umbria: analisi di una casistica decennale
}

\author{
Daniele Crotti', Maria Letizia D'Annibale', Stefano Rossi \\ 'Sezione di Microbiologia e Parassitologia Clinica, Ospedale "R. Silvestrini", Perugia \\ ${ }^{2}$ Laboratorio Analisi Chimico-cliniche e Microbiologiche, Ospedale di Castiglione del Lago (PG)
}

Key words: Strongyloides stercoralis, strongyloidiasis, diagnosis, ipereosinophilia

Strongyloides stercoralis and strongyloidiasis in Umbria, Central Italy: ten years of surveillance.

\section{SUMMARY}

(Strongyloides stercoralis and strongyloidiasis in Umbria: ten years of investigations). The Authors relate prevalences concerning strongyloidiasis in Perugia,Torgiano and Deruta, Provence of Perugia, Region of Umbria, Central Italy, between 1995 and 2004. The specific investigation for Strongyloides stercoralis was initially obtained using Baermann's Method, and after with a modified tecnique of this method and/or with colture on agar of larvae. During 10 years S. stercoralis had been found in 69 elder subjects in relation to 734 examined people (9.4\%) The research of this nematode was usually justified by a periferical hipereosinophilia; infact, among positive people, an increased eosinophilia was documented in $88.4 \%$ of subjects. The Authors describe biological features of this worm as for diagnostical aims as for epidemiological findings, in order to understand the persistence in these moist and temperate areas around rivers and lakes of central Umbria. Thus, the Authors report operating procedures to research and/or to exclude this helminthiaisis in Italian and immigrate population, on the base of anamnestical suggestions of potential infection.

\section{RIASSUNTO}

Gli Autori riportano le prevalenze della strongyloidiasi nel corso del decennio 1995-2004, anno per anno, nell'Umbria centrale, in provincia di Perugia, nei Comuni di Perugia, Torgiano e Deruta. La ricerca mirata di Strongyloides stercoralis fu condotta inizialmente con il Metodo di Baermann, indi con una sua modifica apportata dagli stessi Autori, e/o con la coltura specifica delle larve su agar. S. stercoralis fu complessivamente individuato in 69 soggetti anziani su 734 esaminati specificatamente, pari al 9.4\%. Motivazione precipua alla ricerca di S. stercoralis era data da una ipereosinofilia periferica, sicuramente presente nell' $88.4 \%$ dei soggetti parassitati. Vengono descritte le caratteristiche biologiche di tale nematode sia ai fini diagnostici sia ai fini epidemiologici, anche per comprenderne la verosimile attuale persistenza nella aree descritte a clima caldo-umido della Regione.Vengono così date indicazioni operative per la ricerca o per la esclusione di tale elmintiasi nella popolazione autoctona ed immigrata, sulle base di informazioni anamnestiche probanti una possibile infestazione.

\section{INTRODUZIONE}

Generalità, ciclo biologico e peculiarità clinicodiagnostiche

Strongyloides stercoralis è un elminta appartenente ai nematodi (famiglia Strongyloididae) ed è l'agente responsabile della strongiloidiasi, parassitosi intestinale che può decorrere asintomatica o paucisintomatica, nel sog getto parassitato, anche per moltissimi anni (14).

Tipicamente umana, tale infestazione può colpire anche il cane ed il gatto (28). In alcune regioni dell'Africa sub-sahariana e in Papua Nuova Guinea un'altra specie (tipica dei primati) può infestare l'uomo, S. fülleborni. Altre specie di tale genere infestano esclusivamente altri animali: mammiferi, uccelli, rettili e anfibi (31).

S. stercoralis fu individuato per la prima volta da L. A. Normand nel 1876 e fu descritto, in tale circostanza, come "military worm", in quanto ritrovato da Normand, medico militare all'Ospedale
Navale Francese di Tolone, in un soldato delle truppe coloniali francesi di stanza nella penisola indocinese. Tale soldato rientrava per la precisione dalla Cochin-Cina (l'attuale Vietnam) ed era affetto da una diarrea inarrestabile (17). S. stercoralis fu poi reperito in altri militari di quella truppa, cinque dei quali morirono.

All'autopsia furono reperiti (all'esame microscopico) piccolissimi "vermi tondeggianti" fino allora mai descritti od osservati (di fatto erano larve). Successivamente lo stesso Normand trovò i presunti vermi adulti di tale nematode, ma, non conoscendoli, li inviò al professore di Farmacia J. B. Bavay presso il Consiglio Superiore di Sanità francese, che li chiamò inizialmente Anguillula stercoralis, correlandoli di fatto alle larve in precedenza osservate nei campioni fecali degli stessi pazienti $(8,29,33)$.

Il ciclo vitale di questo nematode è del tutto particolare. L'uomo ne è usualmente l'ospite defini- 
tivo. La femmina parassita partenogenetica (lunga circa $2 \mathrm{~mm}$ ) vive nella mucosa duodenale e dell'intestino tenue (il maschio non svolge alcun ruolo riproduttivo nella fase parassitaria). Le uova embrionate schiudono direttamente nell'intestino e ne escono larve cosiddette rabditoidi (larve di I stadio o L1) che vengono emesse con le feci.

Tali larve (la cui lunghezza è sempre inferiore ai $400 \mu \mathrm{m}$ ) nell'ambiente esterno favorevole (climi caldo-umidi) mutano a larve di II e poi di III stadio (larve filariformi o strongiloidi, L3), le quali possono ripenetrare attraverso la cute di ospiti suscettibili (ciclo diretto). In alternativa, le larve filariformi possono passare alla quarta muta e quindi trasformarsi in femmine e maschi adulti a vita libera. Il ciclo a vita libera è verosimilmente di durata assai lunga, e, in condizioni particolari (non meglio individuate, peraltro), le larve generate da questo accoppiamento sul suolo possono subire una metamorfosi a larve filariformi, capaci di reinfestare un individuo e ritornare ad un ciclo parassitario (ciclo indiretto). In entrambi gli scenari, le larve filariformi L3 (lunghe sempre più di $500 \mu \mathrm{m}$, usualmente attorno a 550-600 $\mu$ ) penetrano la cute integra dell'uomo e migrano attraverso la circolazione linfatica e venosa al cuore destro e poi ai polmoni, e da qui negli alveoli polmonari per risalire l'albero tracheo-bronchiale sino al laringe per essere infine ingerite e raggiungere l'apparato intestinale, ove maturano in femmine adulte che producono uova (38). Il ciclo completo nell'uomo è di circa 1 mese (a volte meno), ovvero: dalla penetrazione transcutanea nell'uomo alla eliminazione delle prime larve rabditoidi (le forme diagnostiche) passano circa quattro settimane (periodo di prepatenza).

Esiste anche l'autoinfestazione, che è tipica di questo elminta (40): alcune larve rabditoidi possono già maturare a larve filariformi in sede intestinale e quindi ripercorrere il percorso cuore destro - polmoni (autoinfestazione interna) oppure maturare in sede perianale e penetrare la cute circostante (con la possibile "sindrome della larva currens") per raggiungere sempre il cuore destro, i polmoni e quindi localizzarsi a livello intestinale (autoinfestazione esterna). Questi processi sono considerati essere il meccanismo con il quale $S$. stercoralis può persistere virtualmente per un tempo indefinito nell'ospite infestato $(19,27)$.

In altri termini, tra parassita e parassitato si instaura un equilibrio tale che permette ad entrambi di sopravvivere senza offendersi od eliminarsi a vicenda. La risposta immunitaria dell'ospite immuno-competente è principalmente mediata dagli eosinofili, per cui spesso l'unico "sintomo" presente è una ipereosinofilia periferica (oltre il $5 \%$, ovvero oltre i 500 eosinofili $/ \mu 1$ ). In presenza di fattori di immuno-compromissione la bilancia si sposta a favore del parassita, per cui compaiono disturbi a livello intestinale $\mathrm{o}$, se già presenti, i medesimi si fanno più importanti e disparati, con diarrea anche grave sino alla possibilità di un ileo paralitico (25).

Nel momento in cui il soggetto fa uso di cortisonici, avviene che $S$. stercoralis riconosce in questi dei metaboliti analoghi presenti in natura necessari alla sua moltiplicazione, e di conseguenza l'equilibrio sino a quel momento più o meno controllato si rompe del tutto e ne consegue una gravissima iperinfestazione con possibilità di exitus per sovrapposte sepsi da Gram-negativi penetrati in circolo dalla sede intestinale con le larve stesse di tale nematode (22).

S. stercoralis è pertanto l'unico nematode (e pressoché l'unico elminta) che reinfesta l'organismo umano nel quale può persistere anche per molti decenni (24); per la sua diagnostica bisogna ricorrere alla ricerca delle larve, e non, come in tutti gli altri casi, delle uova. I comuni sistemi diagnostici utilizzati per tutte le altre enteroparassitosi sono così inadeguati, e bisogna ricorrere a metodi specifici, sensibili e differenziati per la loro evidenziazione $(16,20,21,26,39)$. Tale aspetto è forse quello determinante nella sottostima di tale elmintiasi, anche in Italia, ove invece è tuttora presente (6).

\section{Aspetti epidemiologici}

È stimato che la strongiloidiasi possa affliggere almeno 100 milioni di persone, in circa una settantina di diversi Paesi del Continente, sebbene le stime possano essere ben sotto le reali dimensioni date le peculiarità sopradescritte, soprattutto diagnostiche, di tale nematode (38). S. stercoralis è virtualmente presente in tutte le regioni tropicali e sub-tropicali, in particolar modo nel sud-est asiatico, nell'Africa sub-Sahariana, nell'America Latina $(38,23)$. È peraltro presente anche in regioni a clima temperato: in alcune aree australiane (nella popolazione aborigena), in alcune aree del nord America, in Giappone, e in Paesi del bacino del mediterraneo, quali Spagna, Francia, Grecia ed Italia $(1,5,7,8,34,41)$.

In Umbria, sino alla fine degli anni ' 80 , nulla si sapeva riguardo tale parassitosi. All'inizio degli anni '90 sono stati segnalati i primi casi di strongiloidiasi, in soggetti anziani residenti da sempre, o da giovani, in ambienti rurali $(11,13,35)$. Tale regione è a clima temperato, con estati calde, con la presenza nel suo territorio di pianura di fiumi (Tevere ed affluenti vari, quali Chiascio, Topino e Nestore, nelle valli centrali, non lontano da Perugia), di piccoli corsi d'acqua e di contenute aree lacustri in parte anche acquitrinose, e di un lago (Trasimeno), confinante con la Val di Chiana 
umbra, aretina e senese. Nei periodi invernali ed autunnali tali fiumi, soprattutto nei decenni passati, sono andati incontro a contenute inondazioni delle vallate circostanti, mantenendo così un suolo umido, quantomeno nei periodi primaverili e di inizio estate, adatto al ciclo ambientale a vita libera di S. stercoralis.

L'Umbria è sempre stata regione di lavoro e cultura contadini (36). A dire che, a parte due centri cittadini grossi (di cui uno a impronta operaia, Terni, e l'altro, il capoluogo Perugia, a tradizione impiegatizia, commerciale e turistica), il resto della popolazione viveva in campagna o in piccoli centri abitati strettamente confinanti con aree campestri. I contadini erano così numerosi, soprattutto nel periodo tra le due guerre, ma anche sino agli anni sessanta. Le condizioni economiche, legate ad un rapporto di lavoro sempre dipendente e padronale, erano precarie e sovente vicino all'indigenza (18). I lavori dei campi, manuali sino alla fine degli anni sessanta, imponevano lunghe giornate di lavoro all'aperto, anche lontano da casa. I servizi igienici erano inesistenti ed in ogni caso poco utilizzabili durante le ore lavorative. A parte i periodi più freddi dell'anno, la maggior parte della popolazione, maschi e femmine, bambini ed adulti, camminavano e lavoravano in campagna a piedi scalzi, essenzialmente perché non c'erano i soldi per comprare le scarpe (18, 36). Ne consegue che la defecazione all'aperto e il camminare a piedi nudi per gli stessi terreni rappresentavano ed hanno rappresentato il principale fattore di rischio per l'infestazione da S. stercoralis, il cui esempio più eclatante, in Italia, è stato rappresentato dalle risaie e dal lavoro nelle medesime per i periodi stagionali legati alle varie fasi della coltivazione e della raccolta del riso (37).

Su tali premesse abbiamo voluto raccogliere i dati di dieci anni relativi a siffatta problematica, indubbiamente interessante e delicata, anche al fine di poterne comprendere le reali dimensioni e diffusioni, sia attuali che future.

\section{MATERIALI E METODI}

La ricerca mirata di Strongyloides stercoralis è stata sempre eseguita o con il metodo di Baermann modificato (15), e/o con la coltura delle larve in agar (21). Tale ricerca era sempre affiancata dall'esame coproparassitologico standard, anche per escludere la presenza di altri protozoi e/o elminti (4); a dire che in alcune circostanze anche con l'arricchimento formoloetere/etilacetato (FEA) è stato possibile individuare le larve rabditoidi di tale nematode, sebbene di riferimento fossero i sistemi sopracitati. Inizialmente venne utilizzato anche il metodo di Baermann, successivamente in parte abbandonato a favore dell'altrettanto specifico e sensibile nonché più pratico metodo modificato (15). Nella maggioranza dei casi, purtroppo, per ogni soggetto venne eseguita la ricerca mirata di S. stercoralis su un unico campione fecale, nonostante le indicazioni da noi sempre date (orali o scritte) di procedere sempre agli esami coproparassitologici per tale parassitosi su tre campioni fecali raccolti preferibilmente a giorni alterni (9).

I campioni fecali per la ricerca mirata di S. stercoralis venivano analizzati non appena giunti in laboratorio, in altri termini sempre entro le 12 ore dalla evacuazione. Il metodo di Baermann modificato (o quello originale) era così eseguito e portato a termine in giornata, mentre per le colture delle piastre agarizzate si procedette alla incubazione a $30^{\circ} \mathrm{C}$ per 5 giorni, con osservazioni giornaliere (4).

Utilizzando il metodo di Baermann o la sua modifica l'identificazione delle larve rabditoidi di $S$. stercoralis era ottenuta con la rilevazione delle medesime nei sedimenti fecali, grazie alla osservazione microscopica (10x e 40x), del loro tipico movimento "anguilliforme" e alle caratteristiche morfologiche dopo aggiunta di formalina al 10\%: lunghezza, esofago rabditoide, visibilità dell'abbozzo genitale, come riportato in figura I (34). Utilizzando la coltura su agar, è stato possibile, in alcune circostanze, identificare, oltre alle larve rabditodi (con le caratteristiche di cui sopra), anche larve filariformi o i vermi adulti, maschio e/o femmina rispettivamente, dopo più giorni di incubazione a temperatura di $30^{\circ} \mathrm{C}$ (34). Per le larve filariformi le caratteristiche diagnostiche sono state le seguenti: lunghezza, esofago strongiloide, coda con doppia punta o con punta mozzata, come riportato in figura II (4).

Nel corso del decennio 1995 - 2004 sono stati esaminati specificatamente per la ricerca di $S$. stercoralis i campioni fecali di 734 soggetti, tutti di età superiore ai 55 anni, con una modesta prevalenza dei soggetti di sesso maschile. Si trattava di soggetti italiani, la stragrande maggioranza dei quali erano originari e/o residenti nella provincia di Perugia.

La motivazione alla ricerca mirata di $S$. stercoralis era basata sulla presenza di almeno uno dei seguenti elementi: età superiore ai 65 anni (se non era specificato altro fattore di rischio), ipereosinofilia (in soggetti dai 55 anni in su), disturbi intestinali aspecifici o diarrea importante (in pazienti dai 55 anni in su).

\section{RISULTATI E DISCUSSIONE}

I risultati complessivi sono riportati in tabella 1. Le positività sono state 69 , pari al $9.4 \%$ dei soggetti esaminati. Nel corso del 1995, primo anno di 
studio e di utilizzo di un sistema specifico per la ricerca di $S$. stercoralis, sono stati individuati 7 casi di strongiloidiasi su 73 soggetti esaminati, pari al 9.6\%. Nel corso del quinquennio 1996 2000 le strongiloidiasi sono state 40 , su un totale di 411 soggetti analizzati, pari al 9.7\%.

Nel triennio 2001 - 2003 le strongiloidiasi sono strate 19 su 203, pari al 9.4\% dei soggetti sottoposti all'indagine specifica. Nel corso dell'anno 2004, sono stati esaminati soltanto 47 soggetti e le positività per larve di S. stercoralis sono state 3, pari al $6.4 \%$.

Non abbiamo potuto conservare i dati relativi a tutte le indagini eseguite, per cui alcune informazioni a carattere essenzialmente statistico ci sono sfuggite.

Ad ogni buon conto, nel corso del decennio considerato, a partire dal 1995 per arrivare al 2004 questo è stato il numero di soggetti positivi per $S$. stercoralis: 7 nel 1995, 11 nel 1996, 6 nel 1997, 5 nel 1998, 10 nel 1999, 8 nel 2000, 7 nel 2001, 6 nel 2002, 6 nel 2003 e 3 nel 2004.

Il fatto che i dati siano stati variamente accorpati (anno 1995 e anno 2004, quinquennio 1996-2000, triennio 2001-2003) riflette in parte quanto sopra detto, ma, ad ogni buon conto, non sono emerse differenze significative, tra anno e anno, né in termini di numeri assoluti né di valori percentuali.

I soggetti colpiti sono stati prevalentemente i maschi, 44 maschi su 25 femmine (64\% versus 36\%).

Il range di età dei soggetti con una strongiloidiasi è stato di 57 - 93 anni, con una media di 70 anni ed una mediana di 67 anni.

La maggior parte dei soggetti positivi (tutti autoctoni o, comunque italiani) erano residenti (o lo erano stati) nella provincia di Perugia e sempre in aree rurali, avendo tutti affermato di avere da giovani o giovanissimi camminato "a piedi scalzi" per le campagne della provincia medesima. In particolare 64 pazienti su $69(92.8 \%)$ erano ivi residenti; in 2 casi non venne raccolta la provenienza, mentre nei rimanenti 3 casi i pazienti provenivano (e lì avevano soggiornato in tenera e/o giovane età) da altre province di altre regioni; nella fattispecie in 1 caso dalla provincia di Caserta, in un altro da quella di Reggio Calabria, nel terzo dalla provincia di Messina.

Per quanto riguarda gli aspetti clinici dei soggetti affetti da strongiloidiasi, i dati sono riportati in tabella 2.

Non abbiamo potuto raccogliere o conservare tutte le informazioni al riguardo anche per quanto concerneva i soggetti risultati negativi per una strongiloidiasi. Per quanto riguarda, pertanto e soltanto, i soggetti positivi per S. stercoralis, 61 soggetti su 69, pari all' $88.4 \%$ (ma di alcuni non venne riferito il dato) presentavano una ipereosi- nofilia, variabile tra il $6 \%$ e il $25 \% ; 32$ soggetti presentavano (in molte circostanze in associazione alla ipereosinofilia) disturbi intestinali aspecifici (pari al $46.4 \%$ dei soggetti positivi); in 4 casi $(5.8 \%)$ era presente una diarrea importante, verosimilmente espressione di una iperinfestazione intestinale in fase iniziale (in verità in 2 di questi 4 casi il paziente era in terapia cortisonica per altre concomitanti patologie).

Ovviamente tutti i soggetti avevano confermato che in giovane età avevano camminato per le campagne umbre a piedi scalzi anche ripetutamente nel corso delle stagioni e degli anni, soprattutto in quanto quasi tutti figli di contadini o contadini a loro volta e senza la possibilità economica per acquistare un paio di scarpe protettive. La maggior parte dei soggetti affermò di avere risieduto, allora, lungo le sponde del fiume Tevere nei Comuni di Perugia, Torgiano e Deruta (Media valle del Tevere umbro) o lungo le sponde degli affluenti dello stesso; in rari casi attorno al lago Trasimeno.

A questo proposito riteniamo utile anche riportare i dati relativi alle indagini eseguite presso un'altra struttura diagnostica pubblica sia pur limitatamente agli ultimi quattro anni (2001-2004), quantunque già nel corso del 2000 (12) e degli anni precedenti alcuni casi di strongiloidiasi, nel comprensorio del lago Trasimeno, fossero stati segnalati (35). Ebbene, su 124 ricerche mirate per $S$. stercoralis (con metodo di Baermann modificato e/o coltura specifica), questo fu identificato in 6 casi, pari al $4.8 \%$ del totale. Si trattava di 3 maschi e 3 femmine, tutti di età superiore ai 65 anni, tutti riferenti una ipereosinofilia (tra l' $8 \%$ e il $21 \%$ ), motivo per il quale tale ricerca fu eseguita, seppure in 2 di tali soggetti fossero presenti anche disturbi intestinali con episodi diarroici. Nel dettaglio, S. stercoralis fu osservato nel 7.1\% dei casi analizzati in modo mirato nel 2001 ( $1 \mathrm{su}$ 14), nel 3.6\% nel 2002 (1 caso su 28), mai nel 2003 (su 42 ricerche condotte), nel 10\% nel 2004 ( 4 casi su 40 ). Anche in tale più limitata casistica è stato impossibile recuperare le informazioni complete relative ai soggetti risultati negativi; ad ogni buon conto tali dati confermano la attuale presenza, sia pur a bassa endemia, di tale infestazione nei soggetti anziani residenti, quanto meno da giovani, nel comprensorio di tale lago.

Altre strutture diagnostiche di nosocomi collocati in cittadine nelle Valli medio - alte della Regione (centrali e alta Val Tiberina) hanno, sia pur solo in parte ed in modo assai più contenuto, confermato tale stato di cose; nel senso che alcuni casi di strongiloidiasi, negli ultimi 4 anni, sono stati identificati, nonostante la ricerca delle larve di tale nematode non fosse stata eseguita con meto- 
di di riferimento, ma soltanto con l'arricchimento FEA (4).

La strongiloidiasi è tuttora presente in Umbria, soprattutto nelle valli centrali, caratterizzate dalla presenza di fiumi e laghi attorno ai quali vi sono condizioni ambientali pressoché ottimali per l'esistenza di un ciclo a vita libera di tale nematode. Sicuramente l'endemia è bassa, e in ogni caso una strongiloidiasi in atto è presente soltanto in soggetti anziani e con fattori anamnestici di rischio pregresso per tale parassitosi come esposto nella parte introduttiva.

L'apparente altalenarsi di frequenze più o meno basse nel reperire tale nematode è legato a svariati fattori, seppure nel complesso, nell'arco degli anni, la frequenza media della strongiloidiasi si assesti attorno al 5-10\% dei soggetti con apparente "esposizione" passata alla medesima. Una maggiore attenzione al problema, la scelta di criteri diagnostici adeguati, un maggior numero di soggetti esaminati in generale ed ancor più un maggior numero di soggetti esaminati in modo razionale e mirato, l'esistenza di collaborazione tra curante e paziente da un lato e laboratorista dall'altro, sono tutti fattori che incidono ed hanno inciso sulla variabilità di prevalenze annuali di tale parassitosi.

Purtroppo la ricerca finalizzata di S. stercoralis è ancora da pochissimi eseguita, e questo sicuramente condiziona sia l'epidemiologia sia la reale conoscenza della dimensione del fenomeno, e non soltanto nella popolazione anziana, che in ogni caso si presuppone essere la sola tuttora colpita $(5,6,10,11,15)$.

\section{CONCLUSIONI}

Le conclusioni che si vogliono trarre da tale presentazione sono quelle di raccomandare, quando opportuno, la ricerca mirata anche di S. stercoralis, laddove un'indicazione clinica e/o anamnestica esista e sia significativa al riguardo; tale ricerca mirata deve essere sempre eseguita con uno dei metodi sopra raccomandati, sempre su più campioni, seppure larve rabditoidi di S. stercoralis possano essere evidenziate, più o meno casualmente, anche con il solo arricchimento FEA $(4,10)$.

L'indicazione clinica determinante è, sicuramente, la presenza di una ipereosinofilia periferica, indipendentemente dalla presenza contemporanea di disturbi intestinali (presenti soprattutto in soggetti che abbiano una qualsivoglia forma di immunodepressione), purché in soggetti anziani

Tabella I. Risultati complessivi relativamente alle positività per S. stercoralis nel corso del decennio 1995 - 2004

\begin{tabular}{lccccc}
\hline S. stercoralis & $\begin{array}{c}\text { Anno } \\
1995\end{array}$ & $\begin{array}{c}\text { Quinquennio } \\
1996-2000\end{array}$ & $\begin{array}{c}\text { Triennio } \\
2001-1003\end{array}$ & $\begin{array}{c}\text { Anno } \\
2004\end{array}$ & $\begin{array}{c}\text { Decennio } \\
1995-2004\end{array}$ \\
\hline N. soggetti esaminati & 73 & $4 I 1$ & 203 & 47 & 734 \\
\hline N. soggetti positivi & 7 & 40 & 19 & 3 & 69 \\
\hline percentuale di positività & $9.6 \%$ & $9.7 \%$ & $9.4 \%$ & $6.4 \%$ & $9.4 \%$ \\
\hline
\end{tabular}

Tabella 2. Correlazione dei casi di strongyloidiasi con le caratteristiche cliniche

\begin{tabular}{|c|c|c|c|c|c|c|c|c|c|c|}
\hline \multirow{2}{*}{$\begin{array}{l}\text { straongyloidiasi } \\
\text { Ipereosinofilia }\end{array}$} & \multicolumn{2}{|c|}{1995} & \multicolumn{2}{|c|}{$1996-2000$} & \multicolumn{2}{|c|}{$2001-2003$} & \multicolumn{2}{|c|}{2004} & \multicolumn{2}{|c|}{$1995-2004$} \\
\hline & $5 / 7$ & $71.4 \%$ & $35 / 40$ & $87.5 \%$ & $19 / 19$ & $100 \%$ & $2 / 3$ & $66.7 \%$ & $61 / 69$ & $88.4 \%$ \\
\hline Disturbi intestinali aspecifici & $6 / 7$ & $85.7 \%$ & $18 / 40$ & $45.0 \%$ & $6 / 19$ & $31.6 \%$ & $2 / 3$ & $66.7 \%$ & $32 / 69$ & $46.4 \%$ \\
\hline Diarrea importante & $1 / 7$ & $14.3 \%$ & $2 / 40$ & $5.0 \%$ & $1 / 19$ & $5.3 \%$ & $0 / 3$ & - & $4 / 69$ & $5.8 \%$ \\
\hline
\end{tabular}

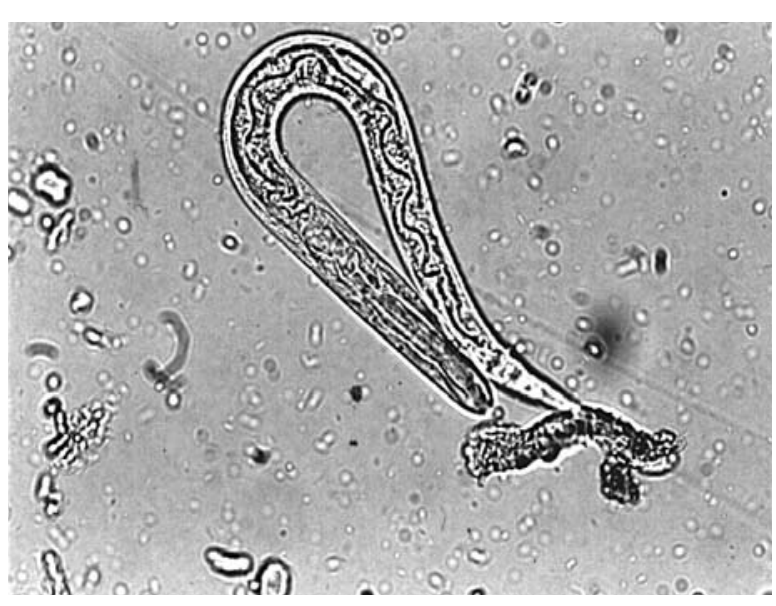

Figura I. Larva rabditoide di S. stercoralis

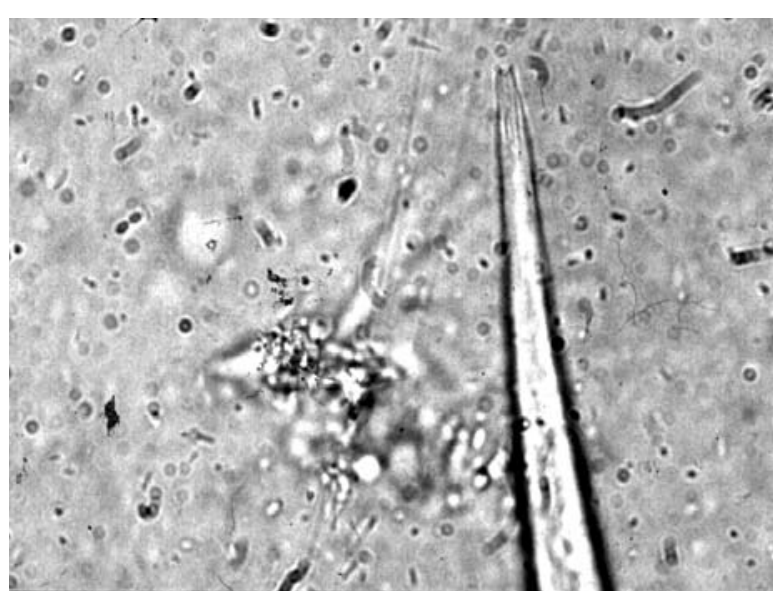

Figura II. Particolare della "coda" di larva filariforme (o strongiloide) di S. stercoralis 
che riferiscano nel passato (lontano o recente) di avere camminato a piedi scalzi su suoli di pianura attorno a corsi o specchi d'acqua ove e che possano così avere contratto tale infestazione.

Così come, in tutti i soggetti che debbano essere sottoposti ad una terapia cortisonica, per i più disparati motivi, è quanto mai opportuno escludere che gli stessi alberghino tale nematode, pena il rischio di gravi complicanze $(2,30,32)$; questo ovviamente sulla base delle informazioni anamnestiche probanti una potenziale esposizione a tale rischio, come in precedenza riportato.

Indubbiamente tale parassitosi precipua dell'anziano dovrebbe scemare nel tempo sino a scomparire.

A dire che ormai l'abitudine o, meglio, la necessità, di camminare a piede nudi nei campi non c'è più. Peraltro tale stato di cose andrebbe verificato, sia nella popolazione autoctona, sia nella popolazione qui migrata da Paesi ove l'endemia della strongiloidiasi è più elevata (3), non tanto per la possibilità di importare lo stesso nematode, qualora si realizzassero eventualmente le condizioni di eliminarlo (esso in fondo è o dovrebbe essere già presente), quanto per la possibilità di perpetuare ciclo endogeno ed esogeno di tale elminta nel caso in cui si ripresentassero abitudini personali o forzate quali quelle esistenti decenni or sono anche nelle nostre campagne: defecazione all'aperto e deambulazione a piedi non protetti; e questo non si potrebbe escludere a priori, vista la tuttora notevole presenza di colture lungo i nostri fiumi e laghi, coltivazioni di tabacco, di cereali e quant'altro e piantagioni di verdure commestibili, ove in vari periodi caldi e caldo-umidi dell'anno molti soggetto vi lavorano, anche per ore e ore, senza possibilità di garanzie igieniche adeguate.

\section{BIBLIOGRAFIA}

1. Adams M, Page W, Speare R. Strongyloidiasis: an issue in Aboriginal communities. Rural and Remore Health. Journal 3 (online) 2003; 152. Available at: http://rrh.deakin.edu.au

2. Barozzi G, Pinelli G, Varani M, et al. Considerazioni cliniche su un caso di iperinfezione da Strongyloides stercoralis. Rass Pat App Respirat 2002; 17: 214-8.

3. Bernieri F, Galli D, Crotti D, et al. Studio Policentrico dell'Associazione Microbiologi Clinici ItalianiComitato di Studio di Parassitologia (AMCLI CoSP) sulla diffusione delle parassitosi intestinali in Italia: parassitosi intestinali nell'immigrato. Giorn It Med Trop 1996; 1(3-4): 97-99.

4. Bernieri F, Crotti D, Galli D, Raglio A. Manuale illustrato di diagnostica parassitologica. Pavia: Selecta Medica; 2001.

5. Bernieri F, Galli D, Giordano S, et al. Indagine nazionale AMCLI-CoSP sulla diffusione delle parassitosi intestinali. Microbiol Med 1996; 11(4): 463-72.

6. Bernieri F, Galli D. Viti F, et al. Parassitosi enteriche autoctone. Microbiol Med 1994; 9(3): 415-8.

7. Casabianca A, Di Matteo L. Strongyloides stercoralis, un problema elmtologico perdurante nella valle del Po: ora anche nell'Astigiano. Parassitologia 1990; 32 (Suppl. 1): 56.

8. Cox FEG. History of Human Parasitology. Clinical Microbiology Reviews 2002; 15(4): 595-612.

9. Crotti D. Parassitosi intestinali autoctone nella seconda metà degli anni '90: considerazioni critiche diagnostiche. Microbiol Med 2002; 17(I): 7-13.

10. Crotti D, D'Annibale ML. Strongyloidiasi nel triennio 2001-2003: considerazioni diagnostiche e clinico-epidemiologiche. GIMMOC 2004; Vol. VIII (N. 1): 1523.

11. Crotti D, Del Sante M. Parassitosi intestinali autoctone nella popolazione del Perugino: prevalenze e considerazioni diagnostico-epidemiologiche. Giorn It Mal Inf 1997; 3(2): 83-8.

12. Crotti D, Rossi S, D’Annibale ML. Strongyloidiasi autoctona in Umbria nel corso del 2000. Microbiol Med 2001; 16(2): 272.

13. Crotti D, Rossi S, Moretti A, et al. Indagine retrospettiva sulle parassitosi intestinali in Umbria. Meeting 1990; 12: 39-40.

14. de Carneri I. Strongyloides stercoralis. In: Parassitologia generale e umana. Casa Editrice Ambrosiana, XI Edizione, Milano 1992; 236-40.

15. Del Sante M, Fonzo G, Crotti D. Infestazioni autoctone da Strongyloides stercoralis: quale diagnostica e quale endemia. Microbiol Med 1997; 12(3): 456-8.

16. Dreyer G, Fernades-Silva E, Alves S, Rocha A, Albuquerque R, Addiss D. Patterns of Detection of Strongyloides stercoralis in Stool Specimens: Implications for Diagnosis and Clinical Trials. J Clin Microbiol 1996; 34(10): 2569-71.

17. Faust EC, Russel PF, Jung RC. Phasmid Nematode Parasites in Man. In: Graig and Faust's Clinical Parasitology. Lea \&Fabiger, VIII Edition, Philadelphia 1979; 284-94.

18. Gatti R. Stanze vuote. Ricordi di una bambina che cresce nell'Umbria contadina di ieri. Arrone (TR): Thyrus; 2003; III Edizione.

19. Gill GV, Beeching NJ, Khoo S, et al. A British Second World War veteran with disseminated strongyloidiasis. Trans R Soc Trop Med Hyg 2004; 98: 382-6.

20. Hernandez-Chavarria F, Avendano L. A Simple Modification of the Baermann Method for Diagnosis of Strongyloidiasis. Mem Inst Oswaldo Cruz 2001; 96(6): 805-7.

21. Jongwutiwes S, Charoenkorn M, Sitthchai P, Akaraborvorn P, Putaporntip C. Increased sensitivity of routine laboratory detection of Strongyloides stercoralis and hookworm by agar-plate culture. Trans $\mathrm{R}$ Soc Trop Med Hyg 1999; 95: 398-400.

22. Keiser PB, Nutman TB. Strongyloides stercoralis in Immunocompromised Population. Clinical Microbiology Reviews 2004; 17(1): 208-17.

23. Machado ER, Costa-Cruz JM. Strongyloides stercoralis and other Enteroparasites in Children at Uberlandia City, State of Minas Gerais, Brazil. Mem Inst Oswaldo Cruz 1998; 93(2): 161-4.

24. Mansfield LS, Niamatali S, Bhopale V, et al. Strongyloides stercoralis: maintenance of exceedingly chronic infections. Am J Trop Med Hyg 1996; 55(6): 617-24.

25. Marques de Oliveira LC, Toffoli Ribeiro C, de Melo Mendes D, Cunha Oliveira T, Costa-Cruz JM. 
Frequency of Strongyloides stercoralis Infection in Alcoholics. Mem Inst Oswaldo Cruz 2002; 97(1): 119-21.

26. Martin-Rabadan P, Munoz P, Palomo J, Bouza E. Strongyloidiasis: the Harada-Mori test rivisited. Clinical Microbiology and Infection 1999; 5(6): 374-6.

27. Neva FA. Biology and Immunology of Human Strongyloidiasis. The Journal of Infectious Diseases 1986; 153(3): 397-407.

28. Nolan TJ. Canine strongyloidiasis. In: Bowman DD (Ed.). Companion and Exotic Animal Parasitology. Ithaca: International Veterinary Information Service (www.ivis.org); 2001.

29. Nolan TJ, Genta RM, Schad GA. Strongyloidosis. In: Palmer SR, Soulsby EJL and Simpson DIH (Ed.). Zoonoses. Oxford University Press, New York 1999; 745-57.

30. Palau LA, Pankey GA. Strongyloides hiperinfection in a renal translant recipient receiving cyclosporine: possible Strongyloides stercoralis transmission by kidney translant. Am J Trop Med Hyg 1997; 57(4): 413-5.

31. Pampiglione S, Canestri Trotti G. Nematelminti. In: Guida allo Studio della Parassitologia. Società Editrice Esculapio, III Edizione, Bologna 1999; 190220.

32. Pasqualini L, Crotti D, Scarponi A, Vaudo G, Mandarino E. Strongyloides stercoralis Infection in a Patient with Crohn's Disease. Eur J Clin Microbiol Infect Dis 1997; 16: 401-3.

33. Petithory JC, Brumpt LC. L'anguillulose opportuniste. Revue francaise des laboratories 1991; 223: 10914.

34. Roman SP, Guzman AP, Guillen SM et al. Edemic strongyloidiasis on the Spanish Mediterranean coast. Q J Med 2001; 94: 357-63.

35. Rossi S, Selvi A, Angori P. Strongyloides stercoralis autoctoni nel comprensorio del lago Trasimeno. Microbiol Med 1992; 7(5): 173-4.

36. Rustici S. Cultura Campagnola, il libro che non c'era. Perugia: Grifo; 2001.

37. Scaglia M, Brusita R, Gatti S et al. Autochtonous strongyloidiasis in Italy: an epidemiological and clinical review of 150 cases. Bull Soc Path Ex 1984; 77(3): 328-32.

38. Siddiqui AA, Berk SL. Strongyloidiasis. Current Treatment Options in Infectious Diseases 2003; 5: 283-9.

39. Siddiqui AA, Berk SL. Diagnosis of Strongyloides stercoralis Infection. CID 2001; 33: 1040-7.

40. Sing A, Leitritz L, Bogner JR, Heesemann J. FirstGlance Diagnosis of Strongyloides stercoralis Autoinfection by Stool Microscopy. J Clin Microbiol 1999; 37(5): 1610-1.

41. Tzanetou K, Geros I, Kalogeropoulou E et al. Is Strongyloides stercoralis endemic in Greece? Arch Hellen Med 2003; 20(1): 60-6.

\section{Daniele Crotti}

Sezione di Microbiologia e

Parassitologia Clinica

Ospedale "R. Silvestrini" - Via G. Dottori 1

06156 S. Andrea delle Fratte, Perugia

Tel. 075 5782218; Fax 0755782404

E-mail: nenedc@tin.it 